\title{
The RNA silencing endonuclease Argonaute 2 mediates specific antiviral immunity in Drosophila melanogaster
}

\author{
Ronald P. van Rij, ${ }^{1}$ Maria-Carla Saleh, ${ }^{1}$ Bassam Berry, ${ }^{2}$ Catherine Foo, ${ }^{1}$ Andrew Houk, ${ }^{1}$ \\ Christophe Antoniewski, ${ }^{2}$ and Raul Andino ${ }^{1,3}$ \\ ${ }^{1}$ Department of Microbiology and Immunology, University of California at San Francisco, San Francisco, California 94143, \\ $\mathrm{USA}^{2}$ Department of Developmental Biology, Centre national de la recherche scientifique (CNRS) URA 2578 Pasteur \\ Institute, Paris 75015, France
}

\begin{abstract}
Most organisms have evolved defense mechanisms to protect themselves from viruses and other pathogens. Arthropods lack the protein-based adaptive immune response found in vertebrates. Here we show that the central catalytic component of the RNA-induced silencing complex (RISC), the nuclease Argonaute 2 (Ago-2), is essential for antiviral defense in adult Drosophila melanogaster. Ago-2-defective flies are hypersensitive to infection with a major fruit fly pathogen, Drosophila C virus (DCV), and with Cricket Paralysis virus (CrPV). Increased mortality in ago-2 mutant flies was associated with a dramatic increase in viral RNA accumulation and virus titers. The physiological significance of this antiviral mechanism is underscored by our finding that DCV encodes a potent suppressor of RNA interference (RNAi). This suppressor binds long double-stranded RNA (dsRNA) and inhibits Dicer-2-mediated processing of dsRNA into short interfering RNA (siRNA), but does not bind short siRNAs or disrupt the microRNA (miRNA) pathway. Based on these results we propose that RNAi is a major antiviral immune defense mechanism in Drosophila.
\end{abstract}

[Keywords: Antiviral defense; RNAi suppressor; dsRNA-binding domain; Drosophila C virus; Cricket Paralysis virus]

Received August 14, 2006; revised version accepted September 15, 2006.

Higher vertebrates rely on both a highly sophisticated "adaptive" immune system that targets pathogens with exquisite specificity as well as a broad-action, nonspecific, "innate" immune response that includes the induction of the nonspecific RNaseL (Silverstein 1989). Intriguingly, invertebrates lack the protein-based adaptive immune response, yet are capable of effectively fighting viral infections. This has raised the question of whether there are additional adaptive and/or innate antiviral mechanisms distinct from the classical mammalian immune system (Brennan and Anderson 2004).

RNA interference (RNAi) is an evolutionarily conserved mechanism for sequence-specific gene silencing guided by double-stranded RNA (Meister and Tuschl 2004). The nonspecific endonuclease Dicer cleaves long, exogenous double-stranded RNA (dsRNA) into short interfering RNA (siRNA), 21- to 25-base-pair (bp) dsRNA with a 2-nucleotide (nt) 3' overhang. These siRNAs are subsequently incorporated in the RNA-induced silencing complex (RISC), where they guide recognition for Argonaute (Ago)-2-mediated cleavage of perfectly com-

${ }^{3}$ Corresponding author.

E-MAIL raul.andino@ucsf.edu; FAX (415) 514-4112.

Article is online at http://www.genesdev.org/cgi/doi/10.1101/gad.1482006. plementary sites in cytoplasmic RNA ("Slicer" activity) (Liu et al. 2004). The related but distinct microRNA (miRNA) pathway uses short, endogenously encoded hairpin RNAs that are sequentially processed into mature miRNAs by two RNases: the nuclear Drosha and the cytoplasmic Dicer. These miRNAs are incorporated into miRISC, which promotes RNA cleavage or translational inhibition upon recognition of cognate target sites in the $3^{\prime}$ untranslated region (UTR) of mRNAs (Bartel 2004).

While miRNAs are known to regulate gene expression essential for proper development and cell physiology (John et al. 2004; Bentwich et al. 2005; Berezikov et al. 2005), the function of the RNAi cleavage pathway in animals remains unclear. Given that the RNA silencing pathway plays a critical role in antiviral defense in plants (Baulcombe 2004; Voinnet 2005), it has been hypothesized that the RNAi cleavage pathway of animals also provides a defense mechanism against parasitic nucleic acids, such as viruses and transposons. Several observations support this hypothesis. Introduction of artificial dsRNA or siRNAs in insects and mammalian cells elicits an effective antiviral response that protects cells against highly lytic viruses (Gitlin et al. 2002; Li et al. 2002; van Rij and Andino 2006). The high specificity of 
antiviral RNAi is highlighted by the observation that resistant viruses readily emerge with single mismatches within the siRNA-target sequence (Gitlin and Andino 2003; Gitlin et al. 2005). Thus, an RNA-based antiviral defense mechanism has the capacity to provide a highly sequence-specific response. However, the observed susceptibility of viruses to synthetic siRNAs does not prove that an antiviral RNAi response is initiated during a natural infection.

Recently, evidence has surfaced supporting the concept that RNAi is involved in the defense against viruses in insects (Keene et al. 2004; Sanchez-Vargas et al. 2004; van Rij and Andino 2006). Components of the RNAi machinery, including Dicer-2 and its cofactor R2D2, were shown to be required for defense of fruit flies against viral infection (Galiana-Arnoux et al. 2006; Wang et al. 2006). Because Dicer is a sequence-nonspecific endonuclease, it is in principle possible that the antiviral function of the RNAi pathway elements relies on cleavage of essential double-stranded intermediates of RNA replication by Dicer, similar to the innate response carried out by RNaseL in mammals. Alternatively, the antiviral activity could rely on the sequence-specific activities of the RNAi response. In this scenario, viral siRNAs produced by Dicer-2 are incorporated into RISC, leading to the specific cleavage of viral mRNAs by RISC and, consequently, suppression of virus infection.

To distinguish between these possibilities, and to test whether RNAi plays a central or an auxiliary role in antiviral defense, we investigate here the role of RISC in the response to viral infection in Drosophila melanogaster. We show that flies defective for the catalytic component of RISC, the nuclease Ago-2, are hypersensitive to infection with Drosophila C virus (DCV) and Cricket Paralysis virus (CrPV), resulting in a 1000 -fold increase in virus production. Thus, Ago-2/Slicer is key to the antiviral response in adult fruit flies. Furthermore, we identified a suppressor of RNAi encoded in the DCV genome, supporting a central role of RNAi in the natural antiviral defense in flies. This suppressor specifically binds long dsRNA, inhibits Dicer processing, and thereby inhibits dsRNA-initiated RNAi. Together, these results provide direct support for the notion that RNAi is central to the natural antiviral immune response in Drosophila.

\section{Results}

The RISC catalytic component Ago2 is required for protection from virus infection

To examine the mechanism of antiviral immunity in the fruit fly we employed Drosophila C virus (DCV), a positive-strand RNA virus from the bicistrovirus family and a major pathogen of Drosophila. Injection of DCV in the abdomen of the fly results in a dose-dependent mortality (Cherry and Perrimon 2004). To evaluate the importance of the insect RNAi machinery in controlling virus replication, we initially studied the effect of a potent viral suppressor of RNAi, the flock house virus B2 protein $(\mathrm{Lu}$ et al. 2005; Sullivan and Ganem 2005). Transgenic flies expressing FHV B2 are defective in RNA silencing elicited by long dsRNA and siRNA, but not in the miRNA pathway (B. Berry and C. Antoniewski, unpubl.). B2 transgenic flies experienced a more rapid course of disease and died faster after inoculation with DCV. Median survival was $2-3 \mathrm{~d}$ shorter in the presence of $\mathrm{B} 2$ than in the control flies (Fig. 1A). Since FHV B2 inhibits dsRNA processing by Dicer (Lu et al. 2005; Sullivan and Ganem 2005), these results suggest that Drosophila Dicer-2 can digest viral dsRNA, consistent with recent reports demonstrating an increase in viral replication in Dicer-2-null mutant flies (Galiana-Arnoux et al. 2006; Wang et al. 2006). Digestion of viral dsRNA could, in principle, account for the antiviral activity of Dicer. Alternatively, Dicer could initiate a more sequence-specific antiviral response, whereby viral siRNAs generated by Dicer-2 are incorporated into RISC, and then used to target singlestranded viral RNA for destruction. If this were the case, we hypothesized that the antiviral activity in flies would be impaired upon loss of RISC function.

A

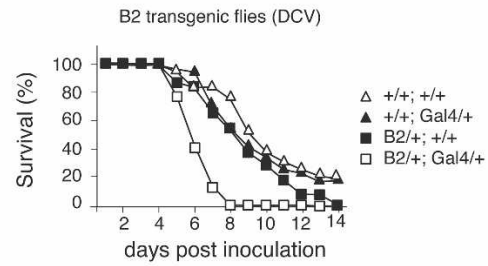

B
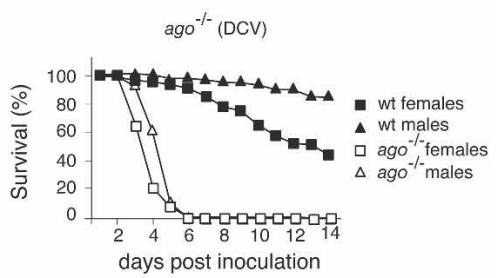

C $\mathrm{der}^{-t^{-}}$or $\mathrm{ago}^{-1-}(\mathrm{CrPV})$

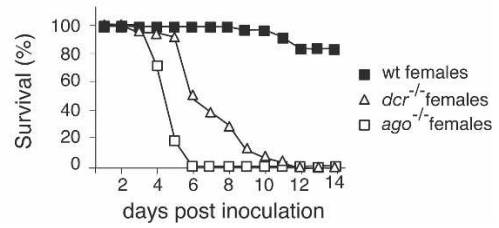

Figure 1. D. melanogaster RNAi mutant flies are hypersensitive to virus infection. (A) Transgenic expression of the flock house virus B2 protein, an inhibitor of Dicer function, increases sensitivity to DCV infection. Flies with the indicated genotypes were injected in the abdomen with $350 \mathrm{TCID}_{50} \mathrm{DCV}$, and survival was monitored daily. $\mathrm{B} 2$ expression (under control of the UASp sequence) was induced by crossing UAS-B2 flies with flies expressing the Gal4 transcription factor from the Daughterless promoter. The F1 progeny from this cross (B2/+; Gal4/+, open squares) expressed B2, whereas B2 expression was not detectable in the control crosses (data not shown). $(B, C)$ Survival of $A g o-$ 2-null male and female mutant flies after virus infection. Homozygous ago- $2^{-/-}, d c r-2^{-/-}$, and wild-type $(\mathrm{wt})$ flies were injected with $350 \mathrm{TCID}_{50} \mathrm{DCV}(B)$ or CrPV $(C)$ and monitored daily for survival. 
To directly establish whether an antiviral response in flies is mediated by RISC we examined the function of the catalytic component of RISC, Ago2, in protection against virus infection. The ago $2^{414}$ mutant flies $\left(\right.$ ago $\left.^{-/-}\right)$ carry a partial deletion of the ago-2 gene, and consequently do not produce Ago2 and are defective in RNAi (Okamura et al. 2004). Strikingly, ago-2 ${ }^{-/-}$and Dicer-2 ${ }^{-/-}$ mutant flies were hypersensitive to virus infection with either of two related bicistroviruses, DCV and CrPV. In Ago2-defective flies, we observed a much earlier onset of disease than in wild-type flies (Fig. 1B,C; Table 1). After injection of $350 \mathrm{TCID}_{50}$ of virus, median survival of ago $^{-/-}$flies was $\sim 5 \mathrm{~d}$ for both viruses, compared with $>14 \mathrm{~d}$ in wild-type flies. Furthermore, disease occurred at lower viral doses. The $50 \%$ lethal dose $\left(\mathrm{LD}_{50}\right)$ in $a g o-2^{-/-}$ or B2 transgenic flies was between 40- and 280-fold lower than in control flies (Table 1). These experiments indicate that RISC activity plays a central role in effective antiviral defense in adult flies.

Increased susceptibility to virus infection in ago-2-/mutant flies is associated with increased levels of viral RNA accumulation and virus titers

There is an important caveat when studying viral sensitivity of flies defective for any components of a major cellular pathway, such as RNAi. Enhanced death following viral infection may be caused by a decrease in fitness or general health of the mutant animal, and not by a direct antiviral activity of the deleted component. Accordingly, we tested whether the increased mortality of ago- $2^{-/}$mutant flies was associated with a direct increase in virus replication (Fig. 2). Even at early time points post-infection, viral titers were 1000-fold higher in ago-2 $2^{-/}$flies compared with wild-type controls (Fig. 2A), indicating that Ago2 indeed plays a direct role suppressing viral replication. The viral titers in wild-type controls during further follow-up never increased beyond $\sim 5 \log _{10}$ TCID $_{50}$ per fly (at days 5,8 , and 11 post-infection), and thus never reached the virus production levels observed in $a g o-2^{-/-}$flies at days 2 and 3 post-infection

Table 1. Lethal viral doses in RNAi-defective flies

\begin{tabular}{lcc}
\hline Drosophila strain & Virus & $\mathrm{LD}_{50}(\mathrm{TCID})^{\mathrm{a}}$ \\
\hline Wild-type & $\mathrm{DCV}$ & 171 \\
ago $^{-/}$ & & 4 \\
$+/+$; Gal4/+ & $\mathrm{DCV}$ & 118 \\
B2/+; +/+ & & 70 \\
B2/+; Gal4/+ & $\mathrm{CrPV}$ & $<21^{\mathrm{b}}$ \\
Wild-type & & 1359 \\
ago $^{-/-}$ & 5 \\
\hline
\end{tabular}

Calculation of LD50 values in each mutant strain using the Reed and Muench method.

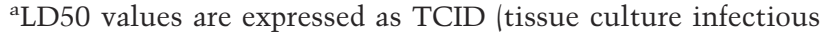
doses).

'In the $\mathrm{B} 2 /+$; Gal4/+ cross, $>50 \%$ of the flies died at the highest dilution tested, and an $\mathrm{LD}_{50}$ value could therefore not be established. This number represents an upper limit of the actual $\mathrm{LD}_{50}$ value.
A

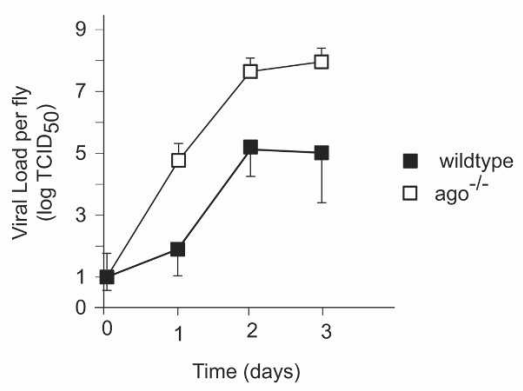

B

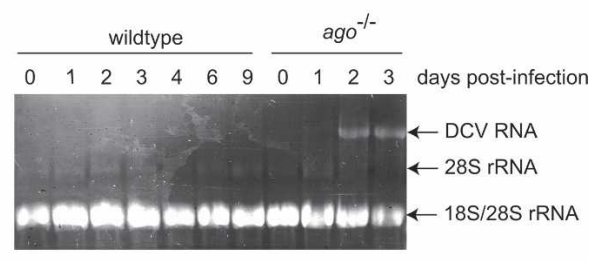

C

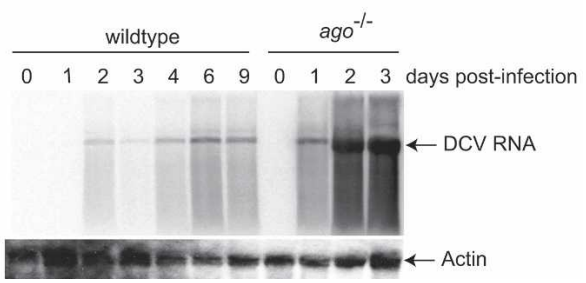

Figure 2. DCV replicates at higher levels in ago-2-null mutant flies. (A) Homozygous ago-2 $2^{-/-}$and wild-type flies were injected with $350 \mathrm{TCID}_{50} \mathrm{DCV}$, and virus production in the flies was monitored over time. At each time point, three pools of five flies were homogenized, and the viral titer in the homogenate was determined by end-point dilution. Titers represent averages and standard deviations of three independent pools of five flies each. $(B, C)$ DCV RNA accumulation is more efficient in $a g O-2^{-/-}$-null mutant flies. ago- $2^{-/-}$and wild-type flies were injected with 350 $\mathrm{TCID}_{50} \mathrm{DCV}$, and 25 flies were harvested daily for detection of viral RNA. (B) Ethidium bromide-stained agarose gel of total RNA preparation. $(C)$ Northern blot analysis of viral RNA. As a loading control, the blots were stripped and rehybridized with a probe specific for actin $42 \mathrm{~A}$. Numbers at the top of the panels indicate days post-infection.

$\left(\sim 8.0 \log _{10} \mathrm{TCID}_{50}\right)$. The increase in viral titers in ago$2^{-/-}$flies was mirrored by a dramatic increase in viral RNA levels (Fig. 2B,C). Indeed, viral RNA in $\mathrm{Ago-} \mathrm{2}^{-/-}$ mutant flies infected with DCV for 2 and $3 \mathrm{~d}$ could be directly detected following ethidium bromide staining of an agarose gel analyzing total RNA. In contrast, in wildtype control flies viral RNA was undetectable even at 9 d post-infection (Fig. 2B). We further confirmed the identity of the RNA bands observed in the ethidium bromide-stained gel by Northern blot analysis (Fig. 2C). While in wild-type flies viral RNA was barely detected before day 6 , in $a g o-2^{-/-}$mutant flies virus RNA was clearly observed at $24 \mathrm{~h}$ post-infection, and by $48 \mathrm{~h}$ virus RNA accumulated at much higher levels than in wildtype control flies (Fig. 2C). We conclude that the enhanced viral susceptibility of $a g o-2^{-/}$flies is due to their inability to control virus replication. The dramatic in- 
crease in viral titers observed when RISC is inactivated establishes ago-2 as a host susceptibility locus for virus infection and provides a direct rationale for the accelerated progression of disease in $a g o-2^{-/-}$flies.

\section{DCV encodes a potent suppressor of the RNAi pathway}

Our results indicate that the RNAi pathway is a central and highly potent antiviral defense mechanism in flies. Given that many plant viruses partially overcome the antiviral RNAi response by encoding suppressors of RNA silencing, we hypothesized that DCV, which is under stringent RNAi control in flies, may have evolved similar RNAi suppressive activities. To determine whether DCV encodes a suppressor of RNAi, Drosophila S2 cells were infected with DCV and, $24 \mathrm{~h}$ later, were cotransfected with a reporter system for RNAi silencing activity, consisting of a Renilla luciferase-expressing plasmid and a specific 541-nt-long dsRNA targeting Renilla mRNA. A transfection control consisting of the unrelated firefly luciferase was included in each experiment. In uninfected control cells, Renilla expression was efficiently silenced (798-fold suppression compared with a control dsRNA). In contrast, silencing was very inefficient in DCV-infected cells (sixfold suppression) (Fig. 3A). Thus, DCV infection somehow inhibits RNAi induced by the 541-nt-long dsRNA. We next examined the mechanism of viral inhibition of RNAi silencing. Because long dsRNA needs to be processed into siRNA by Dicer-2 in order to induce gene silencing, we first determined whether DCV inhibits RNAi by disrupting dsRNA processing. To this end, we repeated the experiment using synthetic 21 -bp siRNA to silence firefly luciferase expression as these short RNAs are directly incorporated into RISC, thus overcoming the need for Dicer processing. In this case, siRNA-induced silencing was not affected by DCV infection (Fig. 3B). To further define the substrate of the DCV RNAi suppressor, we used a panel of dsRNA preparations of different sizes to induce RNAi of the firefly luciferase reporter (Fig. 3C). DCV suppressed RNAi induced by dsRNA ranging from $31 \mathrm{bp}$ up to $592 \mathrm{bp}$ in length, but was unable to inhibit 21-bp siRNA-initiated RNAi; furthermore, suppression of RNAi was more efficient with longer dsRNA preparations (Fig. 3C). These data thus suggest that the DCV suppressor of RNAi acts upstream of RISC, perhaps at the level of dsRNA processing by Dicer-2, which provides the siRNAs for the RNAi silencing pathway. To directly test this possibility, we studied whether virus infection inhibits Dicer-2 activity in cell extracts. We prepared S2 cell extracts from uninfected cells or from cells that were infected with DCV or CrPV 20 h prior to preparation of extracts. In uninfected extracts dsRNA was efficiently processed to 21-bp siRNAs (Fig. 3D, lane 2). In contrast, siRNA production was inhibited in DCVinfected extracts (Fig. 3D, lane 3). Inhibition of Dicer-2 function was specific for DCV, since the closely related CrPV did not affect dsRNA processing (Fig. 3D, lane 4).

Drosophila encodes two Dicer-related genes: Dicer-2,
A

B
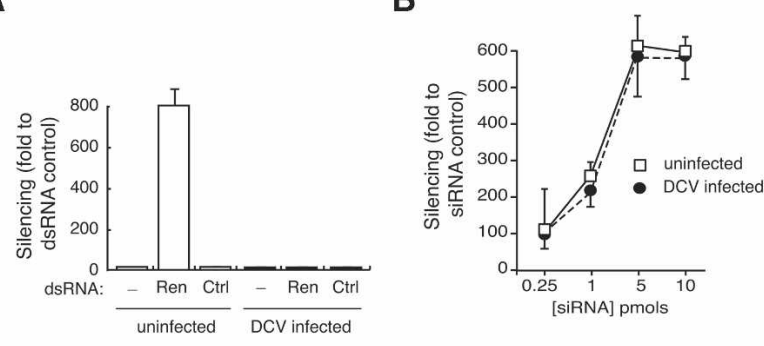

C

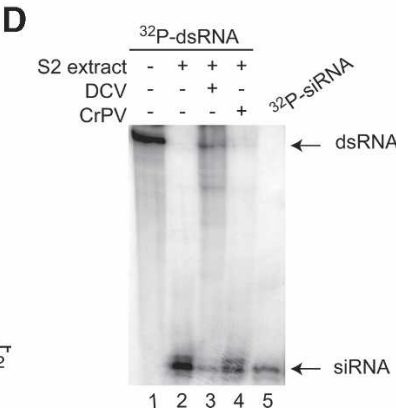

Figure 3. Drosophila C virus encodes a suppressor of long dsRNA-initiated RNAi. (A) Uninfected or DCV-infected Drosophila S2 cells were transfected with expression plasmids encoding firefly and Renilla luciferase and either a 541-bp dsRNA targeting Renilla luciferase or unrelated control dsRNA. Renilla luciferase counts were normalized using firefly luciferase counts, and data are expressed as fold silencing compared with control dsRNA. (B) Experiment was performed as described in $A$, but 21-bp synthetic siRNA targeting firefly luciferase or unrelated control siRNA was cotransfected to initiate RNAi. $(C)$ Experiment was performed as described in A. RNAi was initiated with dsRNAs of 21-592 nt in length, as indicated. Silencing is expressed as percent silencing in DCV-infected over uninfected S2 cells. Data in $A-C$ indicate averages and standard deviations of four independent experiments. $(D)$ Processing of dsRNA in Drosophila S2 cell extract. S2 cells were mock-infected, or infected with DCV or CrPV $20 \mathrm{~h}$ before preparation of cell extracts, at an MOI of 1. Processing of a 116-bp dsRNA into 21-bp siRNA was analyzed on a $12 \%$ denaturing polyacrylamide gel. An end-labeled 21-bp synthetic siRNA was used as a size marker.

involved in the processing of long dsRNA in the RNAi pathway (Lee et al. 2004), and Dicer-1, involved in miRNA biogenesis. To determine whether the suppressor activity encoded by DCV is a general inhibitor of Dicer-like activities, we next examined whether DCV disrupts the miRNA pathway. To this end, we monitored miRNA levels in infected S2 cells over time by Northern blotting (Fig. 4A). The levels of both mature miRNA and the ratio of pre-miRNA to mature miRNA (a processing step that is mediated by Dicer-1) were not changed in infected cells. We further examined whether endogenous miRNAs could assemble into functional RISC in DCV-infected cells. We used a firefly luciferase reporter coding for an mRNA containing two copies of a sequence that is targeted by mature miR $2 b$ at the $3^{\prime} \mathrm{UTR}$ (Fig. 4B, antisense; Saleh et al. 2006). As a control, we used a firefly luciferase mRNA containing these sequences in the reverse orientation (Fig. 4B, sense). S2 cells were cotransfected with these firefly luciferase 
A

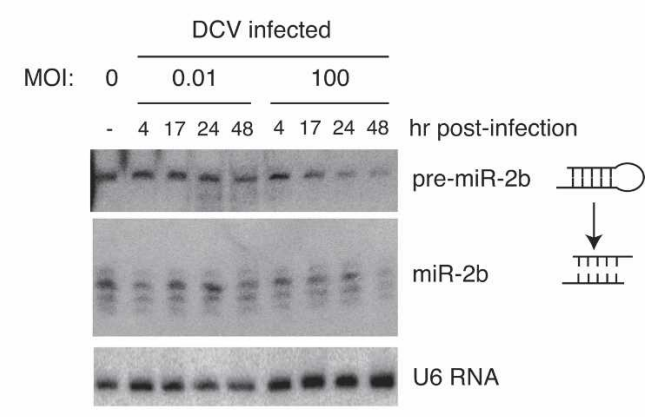

B
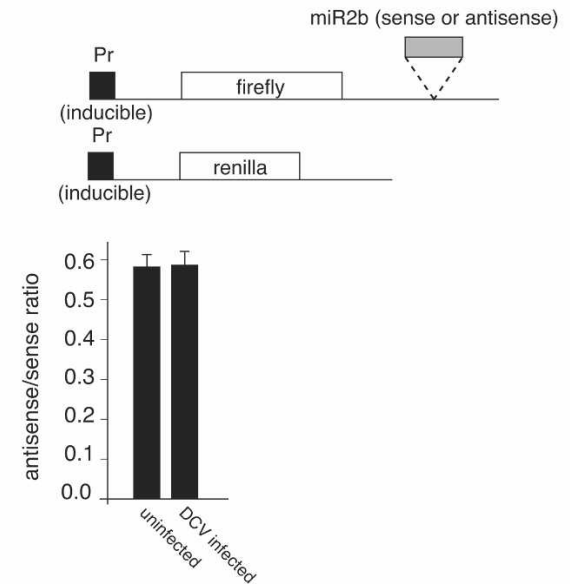

Figure 4. DCV infection does not affect miRNA biogenesis and function. (A) Processed miRNA levels in DCV-infected and uninfected S2 cells. S2 cells were infected with DCV at an MOI of 0.01 and 100, and the presence of miR $2 b$ over time was analyzed by Northern blot. As a loading control, the blot was stripped and rehybridized with a probe specific for U6 snRNA. (B) miR2b is assembled into functional RISC complexes. Uninfected or DCV-infected (MOI of 1) S2 cells were transfected with a plasmid expressing luciferase mRNA containing two copies of a sequence that is perfectly complementary to mature miR $2 \mathrm{~b}$ in either the sense or antisense orientation in the 3'UTR. Luciferase activity was normalized to the expression of a cotransfected Renilla luciferase plasmid that does not contains miR2b complementary sites. Luciferase activity is expressed as the ratio of normalized luciferase counts in luciferase-miR-2 antisense over luciferase-miR-2 sense transfections. Bars indicate averages and standard deviations of four independent experiments.

miR2b reporters as well as with a Renilla luciferase transfection control. Twenty-four hours after transfection, luciferase expression was induced and luciferase activity was monitored. The ratio between firefly luciferase activity of mRNA containing sense and antisense miR2b sequence was virtually identical in infected and uninfected S2 cells (Fig. 4B). Thus, DCV encodes an activity that specifically inhibits RNAi silencing initiated by long dsRNAs but does not affect the miRNA pathway.

The RNAi suppressor is a dsRNA-binding protein encoded at the $N$ terminus of ORF-1

To define the viral factor responsible for RNAi suppression, we examined the amino acid sequence of DCV and identified a canonical dsRNA-binding domain (dsRBD) at the $\mathrm{N}$ terminus of ORF-1 of DCV. We hypothesized that this domain may be responsible for suppression of RNAi, given that many plant virus suppressors of RNAi bind dsRNA (Lakatos et al. 2006; Merai et al. 2006) and our observation that dsRNA processing is inhibited by DCV infection. We subcloned the dsRBD with varying lengths of flanking sequence and tested its ability to suppress RNAi in Drosophila S2 cells (Fig. 5A). As the dsRBD is likely the most $\mathrm{N}$-terminal functional viral gene product, we tentatively refer to this protein as DCV-1A.

Expression of a small fragment of only 99 residues, which include the N-terminal 22 amino acids of ORF-1, the 66-amino-acid minimal dsRBD, and 11 amino acids of C-terminal sequence, was able to efficiently suppress RNAi (Fig. 5B). Increasing the length of the DCV fragment did not further augment the efficiency of suppression of RNAi. dsRBDs are highly conserved and well studied protein domains (Tian et al. 2004; Chang and Ramos 2005). To determine whether DCV-1A suppression requires binding to dsRNA, we mutated highly conserved residues within the dsRBD (L28Y and AA80KK) or residues expected to interact with dsRNA based on available structures (E51A and KK73AA) (Fig. 5C). Three out of four mutations abolished RNAi suppression, including the KK73AA substitution expected to directly interact with dsRNA (Fig. 5D).

To test whether DCV-1A can inhibit RNA silencing in the intact animal, we generated transgenic flies expressing WT-DCV-1A, the inactive KK73AA mutant, or GFP as a control in the developing eye. Efficiency of RNA silencing was monitored in these flies by crossing them to flies in which expression of two mini-white genes was suppressed by expression of a hairpin dsRNA targeting the white gene. Under these conditions the white gene was efficiently silenced, as evidenced by loss of red eye pigmentation resulting in an orange eye color in the GFP control flies (Fig. 5E, GFP). In flies expressing the wildtype DCV-1A, RNA silencing was efficiently suppressed, resulting in expression of the white gene and, accordingly, a red eye color. As expected, in flies expressing the KK73AA DCV-1A mutant, which is unable to suppress RNAi in cell culture (Fig. 5D), the white gene was silenced, albeit less efficiently than in the GFP-transgenic control flies (Fig. 5E). Taken together, these data indicate that the DCV-1A protein acts as a potent RNAi suppressor in flies and confirm that the dsRBD is crucial for the suppressor activity.

\section{$D C V-1 A$ binds long dsRNA with high affinity and inhibits Dicer processing}

To further examine whether DCV-1A suppressor activity is mediated by direct binding to a dsRNA Dicer-2 substrate, we expressed DCV-1A as a fusion to glutathione transferase (WT-DCV-1A) in Escherichia coli and analyzed dsRNA binding by electrophoretic mobility shift assays (EMSA). As a control, we analyzed an inactive mutant suppressor, L28Y-DCV-1A (Fig. 3C). While 
van Rij et al.

Figure 5. Drosophila $\mathrm{C}$ virus dsRBD protein is a suppressor of RNAi. (A) Schematic representation of the DCV genome. The graphic indicates three dsRBD-containing domains that were cloned and examined for suppression of RNAi. (B) Drosophila S2 cells were transfected with plasmids expressing firefly and Renilla luciferase and the indicated dsRBD-containing DCV ORF-1 domains. RNAi was initiated by adding dsRNA specific for firefly luciferase or control dsRNA to the culture supernatant. Data are expressed as fold silencing compared with control unrelated dsRNA. Note that soaking dsRNA is a less efficient method to induce silencing than cotransfecting dsRNA, as was done in Figure 3. (C) Alignment of dsRBD sequences from DCV and different model organisms. Boxed residues indicate residues that directly interact with dsRNA in Xenopus laevis RNA-binding protein A in X-ray structure (Ryter and Schultz 1998). (D) Suppression of RNAi by wild-type and mutant DCV dsRBD in S2 cells. Experiment was performed as described in $B$. Data in $B$ and $D$ indicate averages and standard deviations of four independent experiments. (E) DCV-1A suppresses RNAi in vivo. RNA silencing of the white gene by an inverted repeat (IR [W]) in flies that are transgenic for wild-type $(w \mathrm{t})$ or KK73AA DCV-1A, or GFP as a control. Maximum white gene expression in the absence of RNAi was examined in the GMR-Gal4/+; UASp-GFP/+ control flies that do not express the inverted repeat (left panel, control).
A

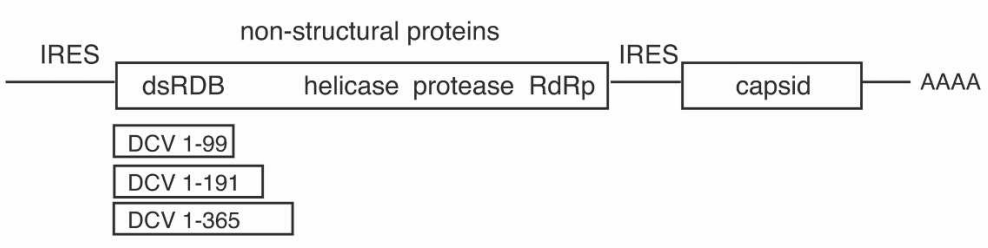

B

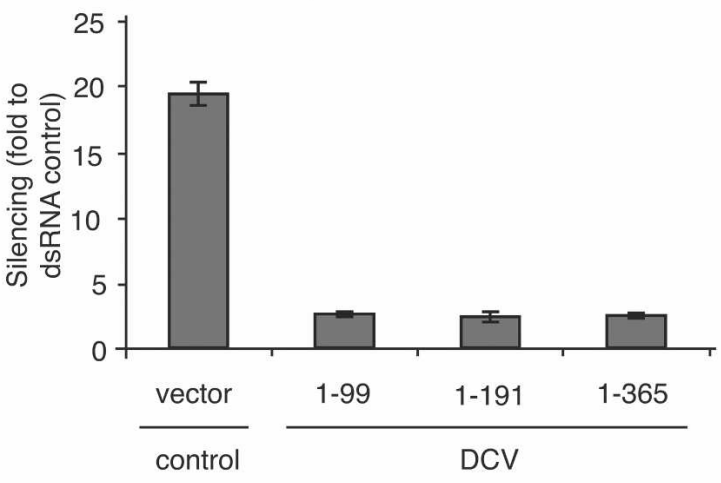

C

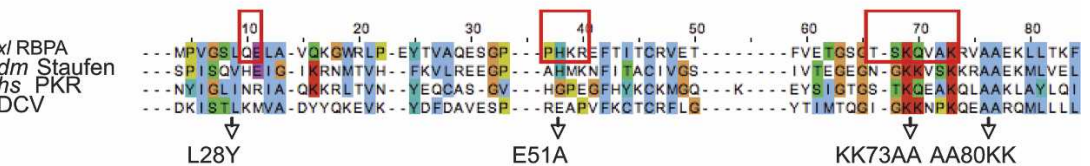

D

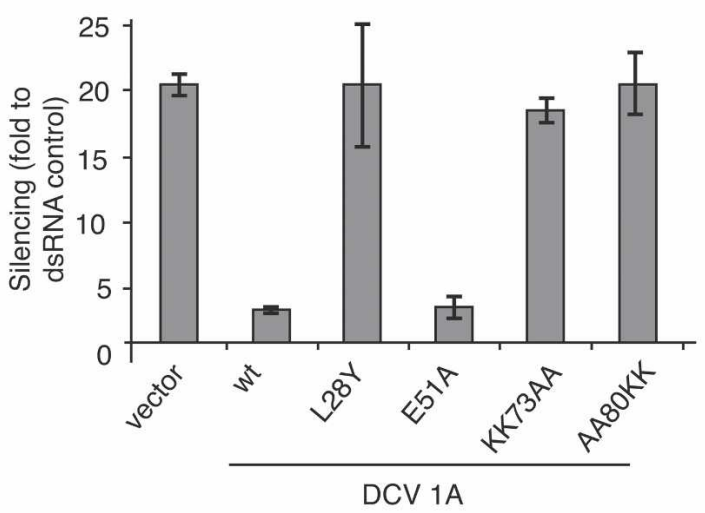

E

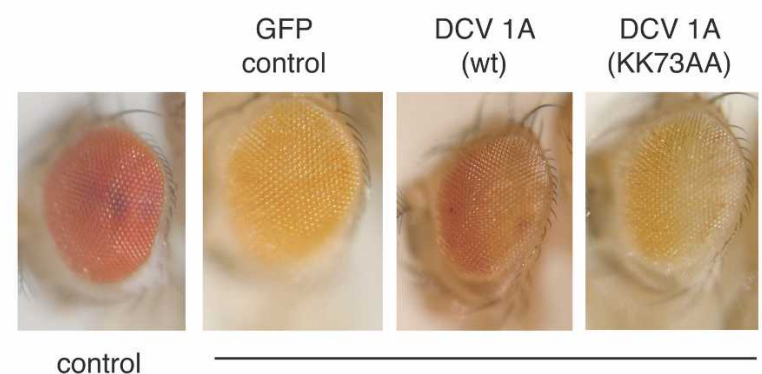

$\operatorname{IR}[\mathrm{W}]$
WT-DCV-1A associated with a 211-bp-long dsRNA probe with high affinity (Fig. 6A,B), the L28Y-DCV-1A mutant was unable to interact with the dsRNA (Fig. 6C, lanes 4-10). With increasing concentrations of WT-DCV$1 \mathrm{~A}$, discrete bands appeared with decreasing mobility, indicating multiple binding events of DCV-1A to a single dsRNA. We observed no binding of ssRNA to DCV-1A (Fig. 6C, lanes 11-18), and DCV-1A bound with similar affinity to a diverse set of dsRNAs, indicating that the interaction is sequence nonspecific (data not shown). In tissue culture, DCV infection inhibited RNAi function elicited by exogenous dsRNA of 31 bp and longer, whereas DCV was unable to prevent siRNA-initiated silencing (Fig. 3B). Furthermore, suppression of RNAi was more efficient with longer, 116- to 592-bp, dsRNA preparations (Fig. 3C). We thus examined whether the binding 
A

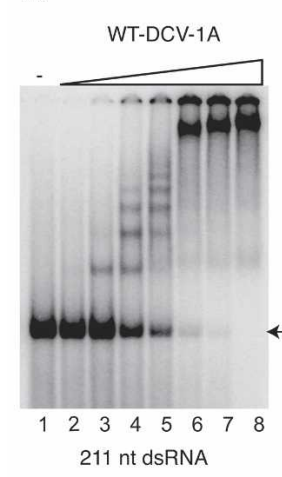

C

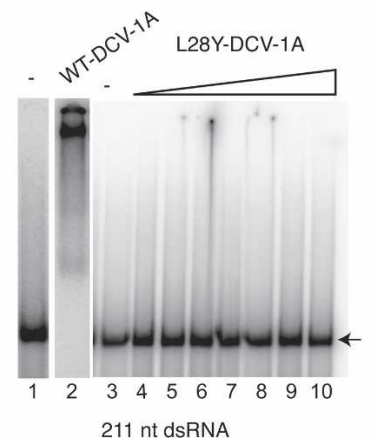

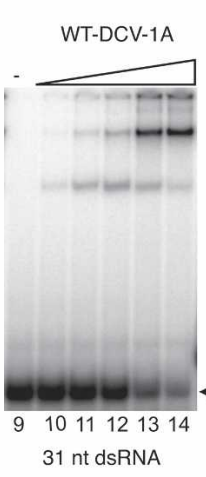

31 nt dsRNA

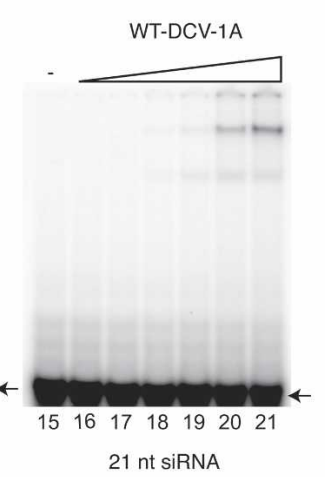

B

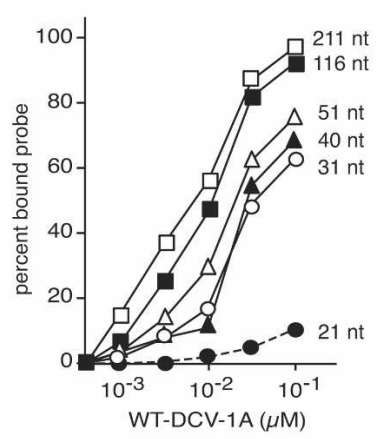

D
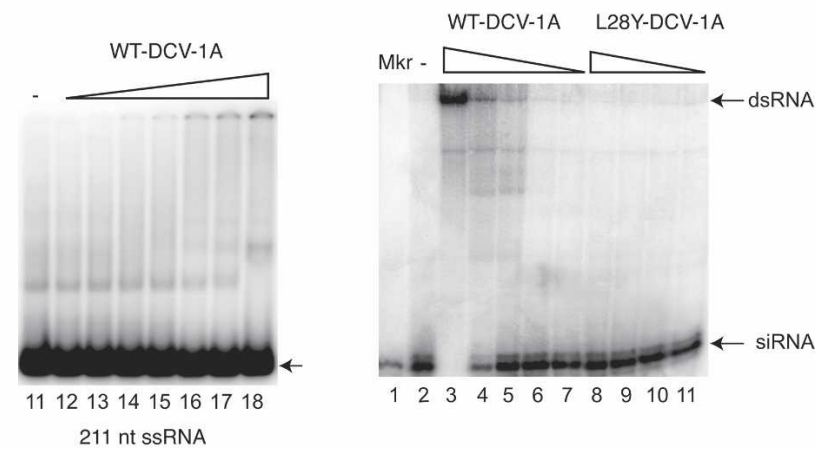

Figure 6. DCV-1A has dsRNA-binding activity and inhibits Dicer processing of dsRNA. (A) RNA mobility shift analyses showing binding of DCV-1A to dsRNA of $211 \mathrm{bp}$ (left panel) and $31 \mathrm{bp}$ (middle panel), and to 21-bp siRNA (right panel). Each probe was incubated with buffer (-) or increasing concentrations of WT-DCV-1A. Concentrations of protein were $0(1 \mathrm{lanes} 1,9,15)$, 0.0001 (lanes 2,16), 0.001 (lanes 3,10,17), 0.005 (lanes 4,11,18), 0.01 (lanes 5,12,19), 0.05 (lanes 6,13,20), 0.1 (lanes 7,14,21), and 0.5 $\mu M($ lane 8). Position of free probe is indicated by arrows. $(B)$ Binding affinity of WT-DCV-1A to dsRNAs of the indicated lengths. The percentage of radiolabeled probe bound by DCV-1A over total probe in mobility shift assays is plotted against WT-DCV-1A concentrations. (C) RNA mobility shift assay of mutant L28Y-DCV-1A to 211-bp dsRNA (left panel) and of WT-DCV-1A to 211-nt ssRNA (right panel). Each probe was incubated with buffer (-) or increasing concentrations of wild-type or mutant DCV-1A. A mobility shift assay of WT-DCV-1A with a 211-bp dsRNA probe was run in parallel as a positive control (lanes 1,2). Concentrations of protein were 0 (lanes 1,3,11), 0.0001 (lanes 4,12), 0.001 (lanes 5,13), 0.005 (lanes 6,14), 0.01 (lanes 7,15), 0.05 (lanes 8,16), 0.1 (lanes 9,17), and 0.5 $\mu M($ lanes 2,10,18). (D) DCV-1A inhibits Dicer processing in S2 cell extracts. Radiolabeled 116-bp dsRNA was processed into siRNA in an S2 cell extract in the presence of increasing concentrations of wild-type and L28Y-DCV-1A or buffer (-). Reaction products were analyzed on a $12 \%$ denaturing polyacrylamide gel. An end-labeled 21-bp synthetic siRNA was used as a size marker (Mkr). Concentrations of DCV-1A protein were 0 (lane 2), 0.5 (lane 3), 0.125 (lanes 4,8), 0.05 (lanes 5,9), 0.005 (lanes 6,10), and $0.00125 \mu M$ (lanes 7,11).

of DCV-1A depends on the size of the dsRNA. WT-DCV1 A does not bind efficiently to 21-bp siRNA, which only formed weak complexes at the highest protein concentration used (Fig. 6A [lanes 15-21], B). In contrast, we observed increasing binding affinity of DCV-1A for dsRNA probes of increasing size (Fig. 6A,B). Thus, the affinity of DCV-1A for the different lengths of dsRNA closely correlates with efficiency of DCV to mediate suppression of RNAi (Fig. 3C).

To establish whether DCV-1A was directly responsible for the observed inhibition of Dicer activity (Fig. $3 \mathrm{D})$, we analyzed processing of dsRNA into siRNA in cell extracts in the presence of recombinant DCV-1A. S2 cell extracts were incubated with dsRNA in the presence of increasing concentrations of wild-type or mutant DCV-1A. In the absence of exogenous viral protein, dsRNA is efficiently processed into 21-bp siRNA by
Dicer-2 (Fig. 6D, lane 2). Addition of wild-type DCV-1A inhibited Dicer activity in a dose-dependent manner (Fig. 6D, lanes 3-7). In contrast, the L28Y DCV-1A mutant had no effect on Dicer activity (Fig. 6D, lanes 8-11). These results indicate that the DCV-encoded RNAi suppressor requires dsRNA-binding activity for inhibition of Dicer-2 function. Furthermore, our biochemical analyses, which indicated that binding of short dsRNAs by the suppressor is very inefficient, explain our observations that DCV is unable to inhibit siRNA- or miRNA-initiated RNAi in vivo.

\section{Discussion}

Our results support the notion that RNA silencing is crucial for antiviral defense in adult $D$. melanogaster. The severity of infection observed in flies lacking a func- 
tional RISC, whereby a few infectious viral particles suffice to rapidly kill the adult animal (Fig. 1; Table 1), argue for a central role of RNAi as an antiviral response. The antiviral mechanism depends on the combined action of Dicer-2 and Ago-2. Dicer-2 senses and cleaves dsRNA, thus generating an siRNA recognition signal that guides RISC for cleavage of viral RNA by Ago-2. In turn, DCV encodes a counterdefense mechanism that consists of a canonical dsRBD that suppresses RNA silencing initiated by long dsRNA, by inhibition of Dicer activity. Because siRNAs are sequence-specific guides for cleavage, this antiviral mechanism could represent the invertebrate homolog of a sequence-specific "adaptive" immune response, in that it would respond to an ongoing infection in a pathogen-specific manner. It remains to be tested whether specific signals persist in the organism for prolonged periods of time, providing something akin to "immunological memory," and whether there are mechanisms for spread of the "immune" antiviral activity throughout the organism.

RNA silencing is instrumental for the local and systemic antiviral response in plants. The RNAi pathway has diversified extensively during evolution in plants. For example, Arabidopsis encodes four Dicer-like genes and 10 Argonaute genes. This diversification may allow plants to efficiently process different dsRNA substrates, and silence gene expression via different effector mechanisms (Baulcombe 2004; Xie et al. 2004). Indeed, there seems to be a differential requirement for two Dicer-like genes, DCL2 and DCL4, for local antiviral silencing and spread of the silencing signal, respectively (Deleris et al. 2006). However, the effector mechanism for antiviral RNA silencing in plants remains undefined. Of the 10 Argonaute family members in Arabidopsis, only Argonaute 1 has thus far been demonstrated to contain Slicer activity, but it does not appear to associate with viral siRNA (Baumberger and Baulcombe 2005). Thus at this stage it is still unclear whether antiviral defense in plants is dependent on a Slicer-dependent RNAi cleavage activity.

Drosophila encodes two Dicer genes and two Argonaute genes, dedicated to either the miRNA pathway (Dicer-1 and Ago-1) or the RNAi cleavage pathway (Dicer-2 and Ago-2) (Lee et al. 2004; Okamura et al. 2004). Our study, together with recent reports (GalianaArnoux et al. 2006; Wang et al. 2006), establish that the antiviral function of RNAi is conserved through evolution, and that antiviral immunity in Drosophila is dependent on a Slicer/Ago-2-mediated effector mechanism. While its reduced genetic complexity makes Drosophila an attractive model system to study the antiviral function of RNAi, many questions remain. For instance, it is unclear what types of RNA structures are recognized by Dicer-2 for the initial cleavage and whether there is a mechanism or feature allowing the RNAi machinery to selectively target viral species but not endogenous dsRNA. A number of observations provide insights into what the physiological substrates for Dicer-2 may be. Our observation that suppression of RNAi mediated by DCV increased in efficiency with increasing length of the trigger dsRNA may imply that the natural viral substrate of Dicer- 2 consists of either the dsRNA replication intermediate or stem loops longer than $30 \mathrm{nt}$. Additional insight comes from a study on the small RNA profile from developing flies (Aravin et al. 2003). The Drosophila stocks used in that study were persistently infected with DCV, and thus 17 small RNAs derived from DCV were cloned. Based on the size profile and the predominant detection of small RNAs from the genomic viral RNA, the authors argued that these small RNAs may not be true Dicer cleavage products (Aravin et al. 2003). A more detailed and systemic analysis of siRNAs in DCV infection is therefore needed to clearly define the Dicer-2 substrate in virus-infected flies.

Another important question will be to determine how viruses escape the inhibitory effect of RNAi. One mechanism, identified in this study, relies on a suppressor with dsRNA-binding activity that inhibits Dicer processing of dsRNA. This mechanism has also been observed in RNA silencing suppressors of plant viruses (Vargason et al. 2003; Ye et al. 2003; Lakatos et al. 2006; Merai et al. 2006), as well as B2 protein from the insect virus Flock house virus (Chao et al. 2005; Lingel et al. 2005; Lu et al. 2005; Sullivan and Ganem 2005). Of note, DCV does not seem to provide a general inhibition of Dicer-2 and Argonaute-2 activity, but rather displays substrate-specific inhibition of RNAi. While RNAi initiated by long dsRNA is inhibited, short siRNA-mediated RNAi is not affected. From an evolutionary perspective, viruses adapt their level of pathogenicity to their hosts to ensure that they can transmit the viral progeny. The presence of an RNAi suppressor may allow the virus to fine-tune the severity of the infection, and may, for example, be crucial to establish persistent infection in natural infections.

We conclude that inhibition of RNAi by dsRNA binding extends beyond the realm of plant viruses, and also occurs in insect viruses. Of note, $\mathrm{CrPV}$, which is closely related to DCV, encodes an RNAi suppressor that maps to the same genomic region as DCV (Wang et al. 2006). However, whereas DCV depends on a canonical dsRBD for RNAi suppression, such a domain could not be predicted in CrPV (and other members of the bicistrovirus family) (R. van Rij and R. Andino, unpubl.). In agreement with this, CrPV did not inhibit dsRNA processing in S2 cells, suggesting a different mechanism of action of the CrPV RNAi suppressor. This suggests independent, convergent evolution of an RNAi suppressor function in otherwise closely related viruses.

\section{Materials and methods}

Cells, plasmids, and viruses

Drosophila S2 cells (Invitrogen) were cultured at $25^{\circ} \mathrm{C}$ in Schneider's Drosophila medium (GIBCO), supplemented with $10 \%$ heat-inactivated fetal calf serum, $2 \mathrm{mM}$ L-glutamine, 100 $\mathrm{U} / \mathrm{mL}$ penicillin, and $100 \mathrm{mg} / \mathrm{mL}$ streptomycin. Firefly (Photinus pyralis) and Renilla reniformis luciferase sequences from the plasmids pGL3 and pRL-CMV (Promega) were cloned into 
pMT/V5-HisB (Invitrogen), generating pMT-Luc and pMT-Ren, allowing copper-inducible expression from a metallothein promoter. cDNA fragments spanning the DCV dsRBD were cloned under the control of the Actin promoter in pAc5/V5-HisB. Mutations in these constructs were introduced using the QuickChange site-directed mutagenesis kit (Stratagene). As a sensor for miRNA biogenesis and function, two copies of a sequence that is perfectly complementary to mature miR2b in either the sense or antisense orientation were cloned in the 3'UTR of the luciferase gene in pMT-Luc, as described (Saleh et al. 2006).

Transfections were performed using Effectene transfection reagent (Qiagen) according to the manufacturer's recommendations. Luciferase expression was assayed using the Dual-Luciferase Reporter Assay System (Promega) and analyzed on a Tecan Ultra-evolution plate reader. DsRNA was generated by in vitro transcription from T7-promoter-flanked PCR products. Synthetic siRNAs were purchased from Dharmacon.

The DCV dsRBD (DCV position 1-99) was cloned into pGex4T.1 (GE Healthcare) for expression as a glutathione Stransferase fusion protein in the E. coli BL21(DE3) strain. The fusion protein was purified using glutathione agarose resin (GST-bind, Novagen) according to the recommendations of the manufacturer, and dialyzed overnight against $20 \mathrm{mM}$ Tris- $\mathrm{HCl}$, $0.5 \mathrm{mM}$ EDTA, $5 \mathrm{mM} \mathrm{MgCl} 2,1 \mathrm{mM}$ DTT, $140 \mathrm{mM} \mathrm{NaCl}, 2.7$ $\mathrm{mM} \mathrm{KCl}$, and $30 \%$ glycerol $(\mathrm{pH}$ 7.9).

DCV and CrPV stocks were prepared on low-passage S2 cells and titered by end-point dilution. Briefly, 25,000 S2 cells per well in a 96-well plate were inoculated with 10-fold dilutions of viral stocks. Cells were transferred to fresh medium at day 7 , and CPE was monitored visually over $14 \mathrm{~d}$. Viral titers were calculated according to the method of Reed and Muench (1938).

\section{RNAi in S2 cells}

The ability of DCV to suppress RNAi was analyzed by silencing luciferase expression in the presence or absence of virus infection. Twenty-four hours prior to transfection, S2 cells were infected with DCV at a multiplicity of infection (MOI) of 1 . These cells were then cotransfected with pMT-GL3 and pMT-Ren, and with dsRNA targeting Renilla luciferase or with synthetic siRNA targeting firefly luciferase. Twenty-four hours after transfection, expression of luciferase was induced by adding $\mathrm{CuSO}_{4}$ to the culture supernatant, and cell lysates were generated after an additional $18 \mathrm{~h}$ incubation. MiRNA function was tested by cotransfection of S2 cells with pMT-Ren with either the miRNA sensor pMT-Gl3-miR2b sense or antisense. The experiment was further performed as described above. The ability of the DCV dsRBD to suppress RNAi was analyzed by cotransfection of pAc-dsRBD with pMT-luc and pMT-Ren. At day 2, RNAi was induced by adding dsRNA targeting firefly luciferase to the culture supernatant. Luciferase expression was induced at $24 \mathrm{~h}$ after feeding dsRNA, and cell lysates were prepared after an additional $18 \mathrm{~h}$ incubation.

\section{Northern blots}

MiRNAs were detected using Northern blot as previously described (Aravin et al. 2003). The blot was hybridized with ${ }^{32} \mathrm{P}$ labeled oligonucleotide antisense to mature miR-2b. To control for equal loading, the blot was stripped and hybridized to a probe complementary to U6 snRNA $\left(5^{\prime}\right.$-TGGAACGCTTCAC GATTTTG-3'). Viral RNA was detected by Northern blot using standard procedures with a random primed DNA probe corresponding to nucleotides 1947-2528 of DCV (accession no. NP 044945).

\section{Fly infections and in vivo RNAi}

Flies were reared on standard medium at $25^{\circ} \mathrm{C}$. Ago- $2^{414}$ and $D c r-2^{\text {L811fsX }}$ flies have been described previously (Lee et al. 2004; Okamura et al. 2004); $\mathrm{w}^{1118}$ flies were used as wild-type controls. UAS-B2 flies will be described in more detail elsewhere (B. Berry and C. Antoniewski, in prep.). Briefly, FHV B2 ORF and wild-type and KK73AA mutant DCV-1A were PCR-amplified and cloned into the pENTR-D-TOPO vector using the pENTR directional TOPO Cloning Kits (Invitrogen), and subsequently transferred into the pUASP vector (Rorth 1998) adapted for the Gateway system (details provided upon request). Transgenic stocks were established using a standard injection procedure. B2 expression was induced by crossing UAS-B2 flies with Daughterless Gal4 flies, and the F1 progeny were challenged with DCV. Two- to three-day-old female flies were injected with 50 $\mathrm{nL}$ of the appropriate virus dilution in $10 \mathrm{mM}$ Tris- $\mathrm{Cl}(\mathrm{pH} 7.5)$ as described previously (Cherry and Perrimon 2004), using a Drummond nanoject injector. Fly mortality at day 1 was attributed to damage invoked by the injection procedure, and these flies were excluded from further analyses. Mortality was monitored daily for $14 \mathrm{~d}$, and every 3-4 d the flies were transferred to fresh food. In all experiments, 40-60 flies per genotypic group were injected. Unless noted otherwise, female flies were used. No significant difference in survival was observed between Ago-2 or wild-type flies after injection of buffer (data not shown). For Northern blots, RNA was isolated from 25 flies using Trizol reagent. Viral titers in the flies were determined by end-point dilution of fly homogenate of three pools of five flies. At the indicated time points, flies were harvested and stored at $-70^{\circ} \mathrm{C}$ until further processing. We confirmed the absence of endogenous virus in fly stocks by titration of uninfected fly homogenate on S2 cells (data not shown). For in vivo RNAi experiments, we established UAS-WT DCV-1A and UASKK73AA DCV-1A transgenic lines in which the DCV suppressor and its variant form are expressed under the control of the GAL4-inducible UAS promoter (Rorth 1998). A P\{GMR-wIR\} transgenic stock (Lee et al. 2004) was recombined by genetic crosses with the $\mathrm{w}^{1118}, \mathrm{P}\{\mathrm{w}+$, GMR-GAL4 $\}$ line to obtain the $\mathrm{w}^{1118}, \mathrm{P}\{\mathrm{GMR}-\mathrm{wIR}\} ; \mathrm{P}\{\mathrm{w}+$, GMR-GAL4 $\}$ homozygous RNAi sensor line. Homozygous $\mathrm{P}\{\mathrm{w}+, \mathrm{pUAS}-\mathrm{Wt}-\mathrm{DCV}\}, \mathrm{P}\{\mathrm{w}+, \mathrm{pUAS}-$ KK73AA-DCV $\}$, or P $\{\mathrm{w}+$, pUAS-GFP $\}$ were crossed to the sensor line, and adult progeny were scored $2 \mathrm{~d}$ after hatching.

\section{Gel mobility shift assays}

Uniformly labeled 116- and 211-bp dsRNA probes were generated by in vitro transcription of sense and antisense sequence in the presence of $\alpha-{ }^{32} \mathrm{P}-\mathrm{UTP}$ by T7 RNA polymerase. The probes were purified from a $6 \%$ denaturing acrylamide gel, and dissolved and annealed in HMK buffer $(200 \mathrm{mM}$ Hepes at pH 7.9, $600 \mathrm{mM} \mathrm{KCl}, 100 \mathrm{mM} \mathrm{MgCl}_{2}$ ). Synthetic 21-bp siRNA and 31-, 40-, 51-, and 116-bp dsRNA, were end-labeled with $\gamma^{-32} \mathrm{P}$ using polynucleotide kinase and purified on a G25 microspin column (GE Healthcare). Probe (1.5 nM) was incubated with varying protein concentrations in the presence of $31.25 \mathrm{mM} \mathrm{DTT}, 0.625$ $\mathrm{mg} / \mathrm{mL}$ yeast tRNA in binding buffer $(5 \mathrm{mM}$ Hepes at $\mathrm{pH} 8.0,25$ $\mathrm{mM} \mathrm{KCl}, 2 \mathrm{mM} \mathrm{MgCl} 2,3.8 \%$ glycerol) for $30 \mathrm{~min}$ at room temperature. Gel shifts of 21-bp siRNA and 31- to 116-bp dsRNA were assayed on $8 \%$ native polyacrylamide gels; gel shifts of 200-bp dsRNA were assayed on 6\% native gels. Gels were run at $4{ }^{\circ} \mathrm{C}$. Radioactivity in mobility shifts was quantified on a Typhoon 9400 Imager (Amersham Biosciences).

\section{Analysis of Dicer activity}

Dicer activity was determined in cell extracts from uninfected and DCV- and CrPV-infected S2 cells. Cells were infected $20 \mathrm{~h}$ 
before preparation of cell extracts, at an MOI of 1 . Cells were washed with PBS, resuspended in lysis buffer (15 mM Hepes/ $\mathrm{KOH}$ at $\mathrm{pH} 7.4,50 \mathrm{mM} \mathrm{KOAc}, 1 \mathrm{mM} \mathrm{Mg}[\mathrm{OAc}]_{2}$, protease inhibitors), and frozen at $-80^{\circ} \mathrm{C}$. Prior to use, extracts were thawed on ice and centrifuged at $14,000 \mathrm{~g}$ for $20 \mathrm{~min}$. The resulting supernatant was tested for Dicer activity for $3 \mathrm{~h}$ at $25^{\circ} \mathrm{C}$, as previously described (Haley et al. 2003), using gel-purified uniformly labeled 116-bp dsRNA. The reaction products were deproteinized by proteinase $\mathrm{K}$ and phenol extraction and analyzed on a $12 \%$ denaturing polyacrylamide gel.

\section{Acknowledgments}

We thank members of the Andino and Pat O'Farrell laboratory for discussions, technical support, and advice on fly work. We thank O. Voinnet and J.L. Imler for helpful discussions. We thank Judith Frydman and Annemieke Jansens for critical reading the manuscript. Peter Sarnow and Sarah Cherry kindly provided DCV and CrPV virus stocks. We are grateful to C. Vaury for the gift of the B2 DNA, and to D. Fagegaltier, L. Röder, E. Georgenthum, and the BestGene, Inc., for generating transgenic flies. Armin Hekele prepared some of the dsRNA used in this study. We thank Richard Carthew for providing the GMR-wIR fly stock, and Phil Zamore for providing fly RNAi mutants and experimental advice on the Dicer assays in S2 cell extracts. B.B. is a Lebanese CNRSL fellow. This work was financially supported by the Pasteur Institute and the CNRS, and by a grant from the ARC (\#3203) to C.A. and NIH grants AI40085 and AI064738 to R.A.

\section{References}

Aravin, A.A., Lagos-Quintana, M., Yalcin, A., Zavolan, M., Marks, D., Snyder, B., Gaasterland, T., Meyer, J., and Tuschl, T. 2003. The small RNA profile during Drosophila melanogaster development. Dev. Cell 5: 337-350.

Bartel, D.P. 2004. MicroRNAs: Genomics, biogenesis, mechanism, and function. Cell 116: 281-297.

Baulcombe, D. 2004. RNA silencing in plants. Nature 431: 356363.

Baumberger, N. and Baulcombe, D.C. 2005. Arabidopsis ARGONAUTE1 is an RNA Slicer that selectively recruits microRNAs and short interfering RNAs. Proc. Natl. Acad. Sci. 102: 11928-11933.

Bentwich, I., Avniel, A., Karov, Y., Aharonov, R., Gilad, S., Barad, O., Barzilai, A., Einat, P., Einav, U., Meiri, E., et al. 2005. Identification of hundreds of conserved and nonconserved human microRNAs. Nat. Genet. 37: 766-770.

Berezikov, E., Guryev, V., van de Belt, J., Wienholds, E., Plasterk, R.H., and Cuppen, E. 2005. Phylogenetic shadowing and computational identification of human microRNA genes. Cell 120: 21-24.

Brennan, C.A. and Anderson, K.V. 2004. Drosophila: The genetics of innate immune recognition and response. Annu. Rev. Immunol. 22: 457-483.

Chang, K.Y. and Ramos, A. 2005. The double-stranded RNAbinding motif, a versatile macromolecular docking platform. FEBS T. 272: 2109-2117.

Chao, J.A., Lee, J.H., Chapados, B.R., Debler, E.W., Schneemann, A., and Williamson, J.R. 2005. Dual modes of RNA-silencing suppression by Flock House virus protein B2. Nat. Struct. Mol. Biol. 12: 952-957.

Cherry, S. and Perrimon, N. 2004. Entry is a rate-limiting step for viral infection in a Drosophila melanogaster model of pathogenesis. Nat. Immunol. 5: 81-87.

Deleris, A., Gallego-Bartolome, J., Bao, J., Kasschau, K.D., Carrington, J.C., and Voinnet, O. 2006. Hierarchical action and inhibition of plant Dicer-like proteins in antiviral defense. Science 313: 68-71.

Galiana-Arnoux, D., Dostert, C., Schneemann, A., Hoffmann, J.A., and Imler, J.L. 2006. Essential function in vivo for Dicer-2 in host defense against RNA viruses in Drosophila. Nat. Immunol. 7: 590-597.

Gitlin, L. and Andino, R. 2003. Nucleic acid-based immune system: The antiviral potential of mammalian RNA silencing. J. Virol. 77: 7159-7165.

Gitlin, L., Karelsky, S., and Andino, R. 2002. Short interfering RNA confers intracellular antiviral immunity in human cells. Nature 418: 430-434.

Gitlin, L., Stone, J.K., and Andino, R. 2005. Poliovirus escape from RNAi: siRNA-target recognition and implications for therapeutic approaches. J. Virol. 79: 1027-1035.

Haley, B., Tang, G., and Zamore, P.D. 2003. In vitro analysis of RNA interference in Drosophila melanogaster. Methods 30: 330-336.

John, B., Enright, A.J., Aravin, A., Tuschl, T., Sander, C., and Marks, D.S. 2004. Human MicroRNA targets. PLoS Biol. 2: e363.

Keene, K.M., Foy, B.D., Sanchez-Vargas, I., Beaty, B.J., Blair, C.D., and Olson, K.E. 2004. RNA interference acts as a natural antiviral response to O'nyong-nyong virus (Alphavirus; Togaviridae) infection of Anopheles gambiae. Proc. Natl. Acad. Sci. 101: 17240-17245.

Lakatos, L., Csorba, T., Pantaleo, V., Chapman, E.J., Carrington, J.C., Liu, Y.P., Dolja, V.V., Calvino, L.F., Lopez-Moya, J.J., and Burgyan, J. 2006. Small RNA binding is a common strategy to suppress RNA silencing by several viral suppressors. EMBO J. 25: 2768-2780.

Lee, Y.S., Nakahara, K., Pham, J.W., Kim, K., He, Z., Sontheimer, E.J., and Carthew, R.W. 2004. Distinct roles for Drosophila Dicer-1 and Dicer-2 in the siRNA/miRNA silencing pathways. Cell 117: 69-81.

Li, H.W., Li, W.X., and Ding, S.W. 2002. Induction and suppression of RNA silencing by an animal virus. Science 296: 1319-1321.

Lingel, A., Simon, B., Izaurralde, E., and Sattler, M. 2005. The structure of the flock house virus B2 protein, a viral suppressor of RNA interference, shows a novel mode of doublestranded RNA recognition. EMBO Rep. 6: 1149-1155.

Liu, J., Carmell, M.A., Rivas, F.V., Marsden, C.G., Thomson, J.M., Song, J.J., Hammond, S.M., Joshua-Tor, L., and Hannon, G.J. 2004. Argonaute2 is the catalytic engine of mammalian RNAi. Science 305: 1437-1441.

Lu, R., Maduro, M., Li, F., Li, H.W., Broitman-Maduro, G., Li, W.X., and Ding, S.W. 2005. Animal virus replication and RNAi-mediated antiviral silencing in Caenorhabditis elegans. Nature 436: 1040-1043.

Meister, G. and Tuschl, T. 2004. Mechanisms of gene silencing by double-stranded RNA. Nature 431: 343-349.

Merai, Z., Kerenyi, Z., Kertesz, S., Magna, M., Lakatos, L., and Silhavy, D. 2006. Double-stranded RNA binding may be a general plant RNA viral strategy to suppress RNA silencing. J. Virol. 80: 5747-5756.

Okamura, K., Ishizuka, A., Siomi, H., and Siomi, M.C. 2004. Distinct roles for Argonaute proteins in small RNA-directed RNA cleavage pathways. Genes \& Dev. 18: 1655-1666.

Reed, L.J. and Muench, H. 1938. A simple method of estimating fifty percent endpoints. Am J. Hyg. 27: 493-497.

Rorth, P. 1998. Gal4 in the Drosophila female germline. Mech. Dev. 78: 113-118. 
Ryter, J.M. and Schultz, S.C. 1998. Molecular basis of doublestranded RNA-protein interactions: Structure of a dsRNAbinding domain complexed with dsRNA. EMBO J. 17: 75057513.

Saleh, M.C., van Rij, R.P., Hekele, A., Gillis, A., Foley, E., O'Farrell, P.H., and Andino, R. 2006. The endocytic pathway mediates cell entry of dsRNA to induce RNAi silencing. Nat. Cell Biol. 8: 793-802.

Sanchez-Vargas, I., Travanty, E.A., Keene, K.M., Franz, A.W., Beaty, B.J., Blair, C.D., and Olson, K.E. 2004. RNA interference, arthropod-borne viruses, and mosquitoes. Virus Res. 102: 65-74.

Silverstein, A.M. 1989. The history of immunology. In Fundamental immunology (ed. W.E. Paul), pp. 21-38. Raven Press, New York.

Sullivan, C. and Ganem, D. 2005. A virus-encoded inhibitor that blocks RNA interference in mammalian cells. J. Virol. 79: 7371-7379.

Tian, B., Bevilacqua, P.C., Diegelman-Parente, A., and Mathews, M.B. 2004. The double-stranded-RNA-binding motif: Interference and much more. Nat. Rev. Mol. Cell Biol. 5: 1013-1023.

van Rij, R.P. and Andino, R. 2006. The silent treatment: RNAi as a defense against virus infection in mammals. Trends Biotechnol. 24: 186-193.

Vargason, J.M., Szittya, G., Burgyan, J., and Tanaka Hall, T.M. 2003. Size selective recognition of siRNA by an RNA silencing suppressor. Cell 115: 799-811.

Voinnet, O. 2005. Induction and suppression of RNA silencing: Insights from viral infections. Nat. Rev. Genet. 6: 206-220.

Wang, X.H., Aliyari, R., Li, W.X., Li, H.W., Kim, K., Carthew, R., Atkinson, P., and Ding, S.W. 2006. RNA interference directs innate immunity against viruses in adult Drosophila. Science 312: 452-454.

Xie, Z., Johansen, L.K., Gustafson, A.M., Kasschau, K.D., Lellis, A.D., Zilberman, D., Jacobsen, S.E., and Carrington, J.C. 2004. Genetic and functional diversification of small RNA pathways in plants. PLOS Biol. 2: E104.

Ye, K., Malinina, L., and Patel, D.J. 2003. Recognition of small interfering RNA by a viral suppressor of RNA silencing. $\mathrm{Na}$ ture 426: 874-878. 


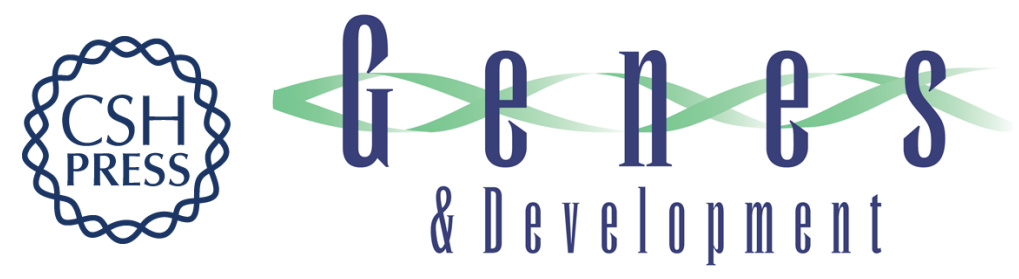

\section{The RNA silencing endonuclease Argonaute 2 mediates specific antiviral immunity in Drosophila melanogaster}

Ronald P. van Rij, Maria-Carla Saleh, Bassam Berry, et al.

Genes Dev. 2006, 20:

Access the most recent version at doi:10.1101/gad.1482006

References This article cites 40 articles, 12 of which can be accessed free at: http://genesdev.cshlp.org/content/20/21/2985.full.html\#ref-list-1

License

Email Alerting Receive free email alerts when new articles cite this article - sign up in the box at the top Service right corner of the article or click here.

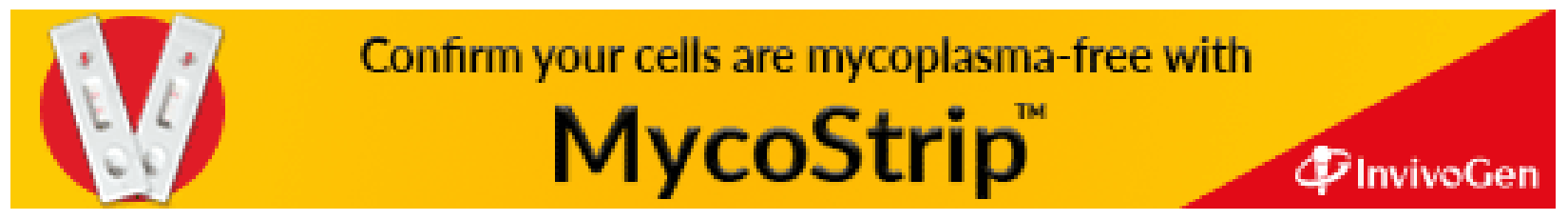

